BULL. AUSTRAL. MATH. SOC.

VOL. $19(1978), 321-324$.

\title{
On the positivity of weak supersolutions of non-uniformly elliptic equations
}

\author{
Neil S. Trudinger
}

\begin{abstract}
The classical meximum principle for homogeneous, second order, uniformly elliptic equations implies that non-negative, classical supersolutions are either positive or vanish identically in the interior of their domain of definition. This paper is concerned with an extension of this result to weak supersolutions of nonuniformly elliptic equations subject to only mild coefficient restrictions.
\end{abstract}

This note is concerned with the positivity of weak non-negative supersolutions of second order elliptic equations subject to only mild coefficient restrictions. Specifically we consider operators of the form

$$
L u=-D_{i}\left(a^{i j} D_{j} u+b^{i} u\right)+c^{i} D_{i} u+d u,
$$

with coefficients $a^{i j}, b^{i}, c^{i}, d(i, j=1, \ldots, n)$, measurable functions on a domain $\Omega$ in euclidean $n$ space $\mathbf{R}^{n}$. We call $L$ elliptic in $\Omega$ if the principal coefficient matrix $A=\left[a^{i j}\right]$ is positive almost everywhere in $\Omega$. Letting $\lambda$ and $\Lambda$ denote the minimum and maximum eigenvalues of $A_{S}$, the symmetric part of $A$, we further assume that $\lambda^{-1}$ and $\Lambda$ are locally integrable in $\Omega$, together with the function $\mu$ defined by

Received 28 August 1978. 


$$
\mu=\alpha_{i j}\left(b^{i} b^{j}+c^{i} c^{j}\right)+|d|,
$$

where $A^{-1}=\left[\alpha_{i j}\right]$ is the inverse of $A$. Finally we assume that there exists a positive constant $K$ such that

$$
\left|a^{i j}(x) \xi_{i} n_{j}\right|^{2} \leq K^{2}\left(a^{i j}(x) \xi_{i j} \xi_{j}\right)\left(a^{i j}(x) n_{i} n_{j}\right)
$$

for all $x \in \Omega, \xi, n \in \mathbf{R}^{n}$. If $A$ is symmetric, inequality (3) with $K=1$ is an immediate consequence of the Cauchy Schwarz inequality. Note that in the expressions (1), (2), and (3), and in what follows, we are employing the standard summation convention that repeated indices indicate summation from $I$ to $n$.

We now have the following theorem.

THEOREM. Let $u$ be a non-negative function in the space $H(A, \mu, \Omega)$ satisfying the inequality, $L u \geq 0$, weakly in $\Omega$. Then either $u$ is positive almost everywhere in $\Omega$ or $u$ vanishes almost everywhere in $\Omega$.

The spaces $H(A, \mu, \Omega)$ are defined in the papers [3], [4]. Under the stricter hypotheses, $\lambda^{-1} \in L^{s}(\Omega), \Lambda, \mu \in L^{t}(\Omega), \frac{1}{s}+\frac{1}{t}<\frac{2}{n}$, Theorem 1 is a consequence of the weak Harnack inequality established in [3]. In this case we can conclude that either $u$ vanishes almost everywhere in $\Omega$ or else

$$
\text { ess inf } u>0
$$

for each domain $\Omega^{\prime}$ strictly contained in $\Omega$. The special case of the above theorem where $A$ is symmetric, $b^{i}=c^{i}=0$ and $d \geq 0$ was proved in [1]. The proof given below which depends on an appropriate choice of test function, is completely different to that in [1].

Proof. The weak inequality, $L u \geq 0$, is equivalent to the integral inequality

$$
\begin{aligned}
L(u, \varphi) & \equiv \int_{\Omega}\left(a^{i j D_{i} \varphi D_{j} u+b^{i} u D_{i} \varphi+c^{i} \varphi D_{i} u+d u \varphi}\right\} \\
& \geq 0 \text { for all } \varphi \geq 0, \varphi \in H^{0}(A, \mu, \Omega) ; \text { (see [3]). }
\end{aligned}
$$


Let us suppose that $u$ vanishes on a subset $E$ of $\Omega$ of positive measure and let $B$ be any ball, strictly contained in $\Omega$, such that $B \cap E$ has positive measure. We then choose as a test function in (4),

$$
\varphi=\frac{\zeta^{2}}{u+\varepsilon}
$$

where $\zeta \in C_{0}^{1}(\Omega), \zeta=1$ on $B$, and $\varepsilon>0$. Since

$$
D_{i} \varphi=\frac{2 \zeta D_{i} \zeta}{u+\varepsilon}-\frac{\zeta^{2} D_{i} u}{(u+\varepsilon)^{2}}
$$

it follows from the local integrability of $\Lambda$ and $\mu$ that

$\varphi \in H^{D}(A, \mu, \Omega)$ and is accordingly a valid test function in (4). We therefore obtain, on substitution,

$$
\int_{\Omega} \frac{\zeta^{2} a^{i j} D_{i} u{ }_{j} u}{(u+\varepsilon)^{2}} \leq \int_{\Omega}\left\{\frac{1}{u+\varepsilon}\left(2 \zeta a^{i j} D_{i} \zeta D_{j} u+2 \zeta b^{i} u D_{i} \zeta+\zeta^{2} c_{i} D_{i} u+\zeta^{2} d u\right)-\frac{\zeta^{2} b_{i} u D_{i} u}{(u+\varepsilon)^{2}}\right\} .
$$

so that, writing

$$
w=\log \left(1+\frac{u}{\varepsilon}\right)
$$

we have, by (3),

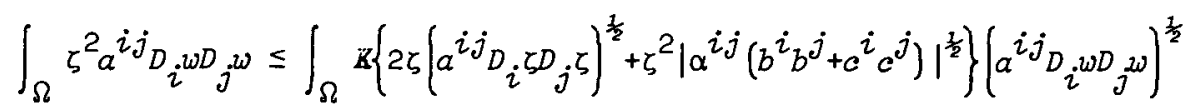

$$
\begin{aligned}
& +\int_{\Omega}\left\{2 K \zeta\left(a^{i j D_{i} \zeta D_{j} \zeta}\right)^{\frac{1}{2}}\left(\alpha_{i j} b^{i} b^{j}\right)^{\frac{1}{2}}+d \zeta^{2}\right\} \text {. }
\end{aligned}
$$

Consequently, by the Schwarz inequality,

$$
\begin{aligned}
\int_{B}|D \omega| & \left.\leq \iint_{B} \lambda^{-1}\right)^{\frac{3}{2}}\left(\int_{B} \lambda|D \omega|^{2}\right)^{\frac{1}{2}} \\
& \left.\leq \iint_{B} \lambda^{-1}\right)^{\frac{1}{2}}\left(\int_{\Omega} \zeta^{2} a^{i j} D_{i} \omega D j\right)^{\frac{1}{2}} \\
& \leq C\left\{\int_{B} \lambda^{-1} \int_{\Omega}\left(\Lambda|D \zeta|^{2}+\mu \zeta^{2}\right)\right\}^{\frac{3}{2}},
\end{aligned}
$$

where $C$ is a constant depending only on $K$. Since $w$ vanishes in $B$ 
on a set of positive measure, we have, by a variant of the Poincaré inequality (see [2, Theorem 6.4]), that

$$
\int_{B} \log \left(1+\frac{u}{\varepsilon}\right)
$$

is bounded independently of $\varepsilon$. Hence by letting $\varepsilon$ tend to zero, we must have $u=0$ almost everywhere in $B$. It then follows that $u=0$ almost everywhere in $\Omega$.

By replacing $u$ by $-u$, we see that a non-positive subsolution of the equation $L u=0$ must either vanish or be negative almost everywhere in $\Omega$. Furthermore if the inequality

$$
d-D_{i} b^{i} \geq 0
$$

holds weakly in $\Omega$, then a non-constant subsolution (supersolution) can only attain a positive maximum (negative minimum) in $\Omega$ on a set of measure zero. The above theorem can thus be viewed as a "weak strong maximum principle". Results of this nature can be applied to the study of eigenfunctions of the operator $L$ (see [1]).

\section{References}

[1] C.V. Coffman, R. Duffin and V.J. Mizel, "Positivity of weak solutions of non-uniformly elliptic equations", Ann. Mat. Pura Appl. (4) 104 (1975), 209-238.

[2] Charles B. Morrey, Jr., Multiple integrals in the caloulus of variations (Die Grundlehren der mathematischen Wissenschaften, 130. Springer-Verlag, Berlin, Heidelberg, New York, 1966).

[3] Neil S. Trudinger, "Linear elliptic operators with measurable coefficients", Ann. Scuola. Norm. Sup. Pisa (3) 27 (1973), 265-308.

[4] Neil S. Trudinger, "Maximum principles for linear, non-uniformly elliptic operators with measurable coefficients", Math. Z. 156 (1977), 291-301.

Department of Pure Mathematics, School of General Studies, Australian National University, Canberra, ACT. 